\title{
High Resolution Spectroscopy of Two Oxygen-Rich SNRs with the Chandra HETG
}

\author{
C.R. Canizares, K.A. Flanagan, D.S. Davis, D. Dewey, J.C. Houck, \\ M.L. Schattenburg \\ Center for Space Research, Massachusetts Institute of Technology \\ Cambridge, MA 02139
}

\begin{abstract}
The HETG can be used to obtain spatially resolved spectra of moderately extended sources. We present preliminary results for two well studied, oxygen rich supernova remnants in the Magellanic clouds, E0102-72 and N132D. The dispersed spectrum of E0102-72 shows images of the remnant in the light of individual emission lines from $\mathrm{H}$-like and He-like ions of $\mathrm{O}, \mathrm{Mg}, \mathrm{Ne}$ and He-like $\mathrm{Si}$ with no evidence of Fe. The diameters of the images for various ions, measured in the cross-dispersion direction, increase monotonically with the ionization age for the given ion. This shows in detail the progression of the reverse shock through the expanding stellar ejecta. We see clear evidence for asymmetric Doppler shifts across E0102-72 of $\sim \pm 2000 \mathrm{~km} \mathrm{~s}^{-1}$. These can be modelled approximately by a partially-filled, expanding shell inclined to the line of sight. The dispersed spectrum of N132D is more affected by spatial/spectral overlap but also shows monochromatic images in several strong lines. Preliminary spectra have been extracted for several bright knots. Some regions of oxygen-rich material, presumably stellar ejecta, are clearly identified. Additional details on E0102-72 are presented by Flanagan et al. and Davis et al. in these proceedings, and further analysis is in progress.
\end{abstract}

\section{INTRODUCTION}

The High Energy Transmission Grating (HETG) consists of an array of periodic nanostructures that can be inserted behind the Chandra mirrors in order to disperse the focused X-ray beam into a spectrum at the focal plane. The HETG array includes two grating types, MEG and HEG, which together cover the energy band 0.4-8 keV with resolving powers of up to 1000 . The dispersed spectrum is read out by the Advanced CCD Imaging Spectrometer (ACIS-S). A complete description is given in [1] and details can be found in the Chandra Proposers guide and at http://space.mit.edu/HETG.

As a "slitless" dispersive spectrometer, the HETG is most straightforward when used to observe point sources: a zeroth order image is formed at the normal focal 
point, with dispersed spectra on either side (the HEG and MEG spectra are offset from one another by 10 degrees to form a shallow "X" centered on zeroth order). For point sources, the resolution of the complete spectrometer is $0.022 \AA$ and $0.012 \AA$ for MEG and HEG, respectively. For moderately extended sources, the spatial and spectral information are mixed in the dispersion direction, but not in the cross dispersion direction. Although this mixing complicates the analysis, moderately extended sources like those presented here are very amenable to simultaneous spectral/spatial analysis, particularly if their emission is dominated by distinct spectral lines. In that case the dispersed spectrum is analogous to a spectro-heliogram, showing a series of monochromatic images of the source in the light of individual spectral lines.

This paper gives a brief report of HETG observations of two relatively compact supernova remnants (SNRs) in the Magellanic clouds, E0102-72 and N132D. Both are members of the oxygen-rich class of SNRs, of which Cas A is often taken as the prototype and which are thought to be products of Type 1b or II supernovae in massive stars [2]. More detailed reports of our results on E0102-72 are included in the proceedings of this meeting (see [3] [4]).

\section{E0102-72}

The SNR 1E0102.2-7219 is a well studied member of the oxygen rich class of supernova remnants located in the SMC. It has a radius of 20 arc sec $(6.4 \mathrm{pc})$, which is a perfect size for spectral imaging with the HETG. Recently, moderate resolution X-ray spectra integrated over the remnant were obtained with ASCA [5]. Gaetz et al. [6] reported spectrally resolved imaging from Chandra's ACIS detector, which shows an almost classic, text-book SNR with a hotter outer ring identified with the forward shock surrounding a cooler, denser inner ring which is presumably the reverse-shocked stellar ejecta. Hughes et al. [7] combined the Chandra image with earlier Einstein and ROSAT images to measure X-ray proper motions, which give an expansion age of $1000 \mathrm{yr}$, consistent with earlier estimates (they also deduce that a significant fraction of the shock energy has gone into cosmic rays). Blair et al. [2] analyzed extensive observations with HST of the optical/UV filaments. At this meeting, Eriksen et al. [8] report the analysis of new Fabry-Perot observations that yield an age of $\sim 2100$ yr for the remnant.

We observed E0102 for a total of $140 \mathrm{ksec}$ on two occasions with the Chandra HETG. A portion of the dispersed spectrum is shown in Fig. 1. It shows multiple images of the SNR in the light of individual spectral lines from various ions. Most prominent are the lines of $\mathrm{H}$-like and He-like $\mathrm{O}, \mathrm{Ne}, \mathrm{Mg}$. We see no clear evidence for any Fe emission (although weak Fe lines are reported by Rasmussen et al. [9] at this meeting from an observation with the XMM-Newton Reflection Grating

Spectrometer). The O VIII image alone suggests that this remnant contains at least several solar masses of oxygen in the reverse-shocked ejecta. 


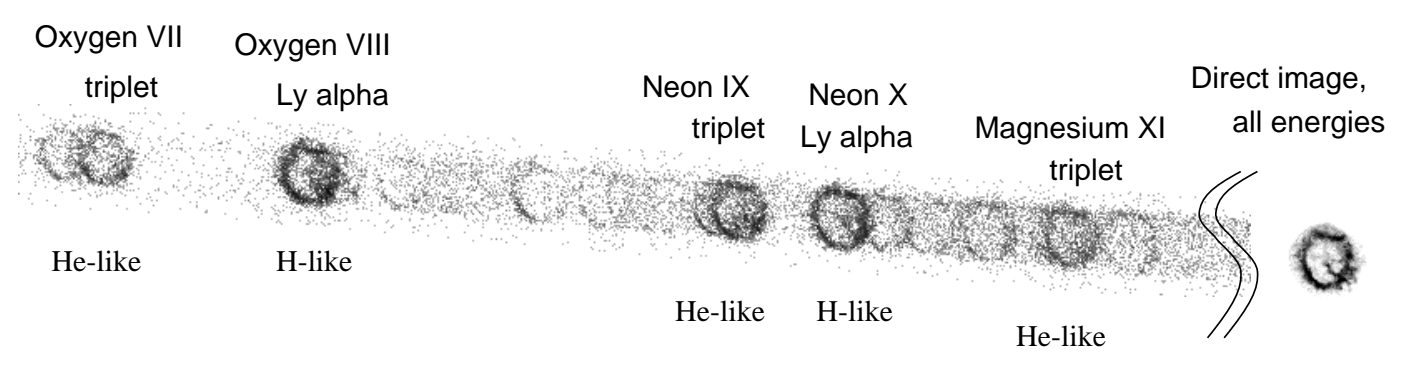

FIGURE 1. Dispersed high resolution X-ray spectrum of E0102-72. Shown here is a portion of the -1 order formed by the medium energy gratings (MEG). The zeroth order, which combines all energies in an undispersed image is at the right. Images formed in the light of several strong $\mathrm{X}$-ray emission lines are labeled.

\section{Imaging the Reverse Shock}

The time scale for the ions in E0102 to reach ionization equilibrium is comparable to or longer than its age, so one would expect the plasma not to have achieved ionization equilbrium [10]. Hayashi et al. [5] found evidence for departures from ionization equilibrium for the integrated spectrum of the SNR (which ASCA could not resolve spatially) based on the relative intensities of He-like and H-like lines. They could not find a consistent model to explain the line ratios for different ions, even allowing for multiple components, and concluded that abundance inhomogeneities must be present. If we integrate the flux over the remnant for several of the strong, relatively isolated lines, we also find line ratios that indicate departures from ionization equilibrium [4].

Gaetz et al. [6] find direct evidence for progressive ionization of oxygen in the spectrally resolved Chandra image. They compared monochromatic images (at CCD spectral resolution) at the energies of the O VII and O VIII lines, and found that the $\mathrm{O}$ VII emitting region lies inside that of $\mathrm{O}$ VIII. This is what one would expect if the ejecta are being subject to a reverse shock propagating backwards (in a Lagrangian sense) towards the center of the remnant (ionization at smaller radii lags behind the ionization at larger radii).

We observe this progressive ionization quantitatively by comparing the images from the He-like resonance lines and H-like Lya lines of $\mathrm{O}$, Ne and $\mathrm{Mg}$, and of He-like Si. Details are given elsewhere in these proceedings [3], but the qualitative evidence can be seen directly from Fig 1 . For both $\mathrm{O}$ and Ne, the images of the Helike lines are noticeably smaller than the H-like images. Cuts across the spectrum in the cross-dispersion direction (to avoid any spectral/spatial confusion) show that, for each ion, the diameter of the He-like image is smaller than that of the H-like image. Furthermore, for a given temperature, one can find a characteristic ionization time scale or ionization age (ionization age is given by $\tau=n_{e} t$ for electron density $n_{e}$ and time $t$ ) at which a given ion reaches its peak population fraction, and therefore its peak emissivity in an ionizing plasma. Doing this for the ions in question (assuming $\log \mathrm{T}=7.05$ ) shows a monotonic increase in the diameter of the 
emitting region with increasing characteristic ionization timescale This suggests a simple interpretation, in which the differences in image diameters for the different ions are caused entirely by the progressive passage of the reverse shock through relatively homogeneous stellar ejecta. A more comprehensive analysis, which will take into account the likely density gradient in the ejecta, for example, is now underway.

\section{Differential Doppler Shifts}

We have found clear evidence for Doppler shifts in several of the emission lines, and evidence that these vary systematically across the remnant. This can be seen from a comparison of the two (plus and minus order) dispersed images from a strong line, such as Ne $\mathrm{x}$ Ly $\alpha$, with each other and with the zeroth order image (constructed by selecting a narrow range of ACIS pulse heights at the corresponding line energy). For example, even in Fig. 1 it is clear that the Ne $\mathrm{x}$ image appears slightly elongated in the dispersion direction compared to the zeroth order image.

The fact that the HETG has both plus and minus order images breaks the spectral/spatial degeneracy that would otherwise confuse the signature of a Doppler shift. A dispersed image in the light of a single line that is distorted due to intrinsic spatial variations will look identical on either side of zeroth order, whereas a distortion due to a wavelength (Doppler) shift will appear with opposite offsets in the plus and minus orders, (i.e. a shift to longer wavelength moves to the right in the plus order but to the left in the minus order image, showing reflectional symmetry about zeroth order). Furthermore, comparison of plus and minus orders also allows one to identify and avoid confusion from the overlapping images of nearby lines.

We have examined the relatively clean dispersed $\pm 1^{\text {st }}$ order images in the $\mathrm{Ne}$ $\mathrm{x} \operatorname{Ly} \alpha$ line with the corresponding zeroth order image and find clear evidence for distortions with the mirror symmetry expected for Doppler shifts in wavelength (some positive, others negative). More than a dozen bright regions around the remnant were analyzed to determine the sense and magnitude of the shifts.

The result of the Doppler analysis for Ne $\mathrm{x}$ Ly $\alpha$ is shown in Fig. 2, where the arrows pointing left indicate blue-shifts and those pointing right indicate redshifts. The magnitudes of the shifts range from -1600 to $+2300 \mathrm{~km} \mathrm{~s}^{-1}$. The velocity structure in the X-ray is systematic and clearly asymmetric, showing only redshifts on the eastern side but both red and blue shifts on the western side. The velocities measured for some of the optical knots, which generally lie interior to the brightest portions of the X-ray bright ejecta, are comparable to those found here and also show complex, asymmetric structure [11]

We have made a preliminary effort at modeling the asymmetric velocity structure shown in Fig 2. Rough correspondence is achieved if the emission comes from a partially filled spherical shell with azimuthal symmetry whose axis is inclined to the line-of-sight. The inner radius of the shell is $4.3 \mathrm{pc}$, its thickness is $1.1 \mathrm{pc}$, and the velocity increases with radius. The emissivity is concentrated toward the 


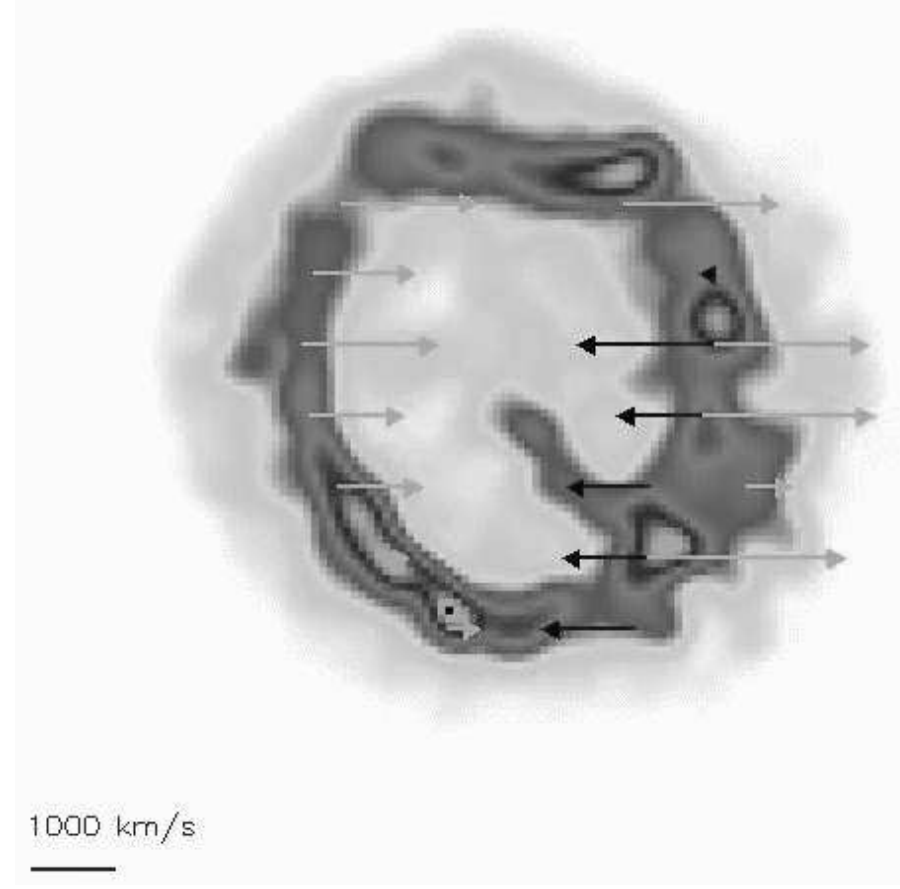

FIGURE 2. Doppler velocities of E0102-72 Ne x Ly $\alpha$. The lengths and locations of the arrows indicate the relative velocities and approximate locations of red/blue shifted ejecta. Arrows pointing left represent blue shifts; arrows pointing right represent red shifts.

equatorial plane (we assume a Gaussian distribution in the cosine of the latitude on the spherical shell with $\sigma=0.35$ ). Interestingly, Hughes [13] deduced that the surface brightness distribution measured with the ROSAT HRI also indicated that the emission was concentrated in a thick ring rather than a spherical shell.

\section{N132D}

N132D is located in the LMC at a distance of $50 \mathrm{kpc}$, with a radius of $\sim 50$ arc sec $(\sim 12 \mathrm{pc})$ and an estimated age of $3000 \mathrm{yr}$. Several recent X-ray studies have been presented from Beppo-SAX [14] and ASCA [15]. Previously, Hwang et al. [16] performed a non-equilibrium ionization analysis using moderate resolution spectra from the Solid State Spectrometer combined with high resolution spectroscopy of selected lines obtained with the Focal Plane Crystal spectrometer (both instruments were on the Einstein observatory). All these studies suggest the overall elemental abundances for N132D are roughly similar to those for the LMC as a whole. This indicates that $\mathrm{N} 132 \mathrm{D}$ is dominated by swept-up material rather than ejecta, and is thus at a later stage of evolution than E0102-72. The most recent optical/UV 
data [2] support this conclusion.

We observed N132D for $100 \mathrm{ksec}$ with the Chandra HETG with a roll angle that places the dispersion direction parallel to the narrow axis of this U-shaped SNR. A portion of the MEG spectrum is shown in Fig 3. The combination of larger size and more numerous emission lines in N132D result in greater spectral/spatial overlap than in E0102-72. Nevertheless, we can distinguish clear monochromatic images in the light of individual emission lines of oxygen, neon and iron, for example (iron is largely responsible for the enhanced number of lines). Even a preliminary examination indicates strong spatial variations in the concentrations of these species, with some features richer in iron and others in oxygen. The fact that we see oxygen rich material is particularly significant, since, contrary to the situation in E0102, no direct X-ray evidence for oxygen rich ejecta had previously been seen [15] (Behar et al. [17] also see regions of enhanced oxygen with XMM-Newton)

We have begun to measure the spectra of selected knots in N312D by extracting slices through the dispersed spectral image centered on the specific features of interest. Typically, a given feature will have some "tilt" with respect to the dispersion direction, so spectra in narrow slices perpedicular to the dispersion direction are shifted to a common origin (e.g. a stack of slices through the dispersed images of a curved feature whose zeroth order is shaped like '(' is shifted slice-by-slice and aligned so that its zeroth order becomes a straight line perpendicular to the dispersion direction). The stacked, shifted slices of dispersed spectra are then projected

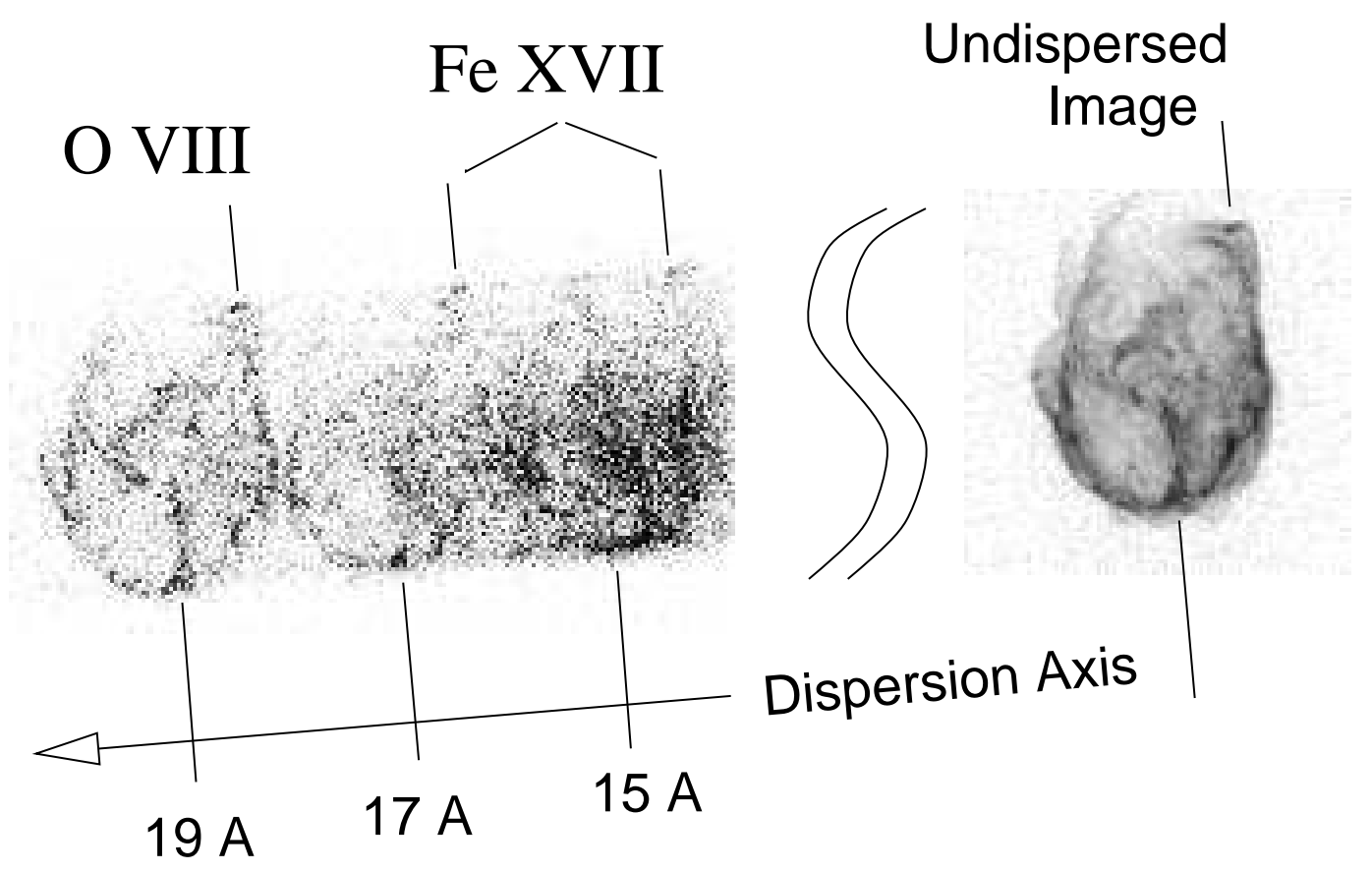

FIGURE 3. Dispersed high resolution X-ray spectrum of N132D. A portion of the MEG spectrum is shown at left, with zeroth order image at right. 

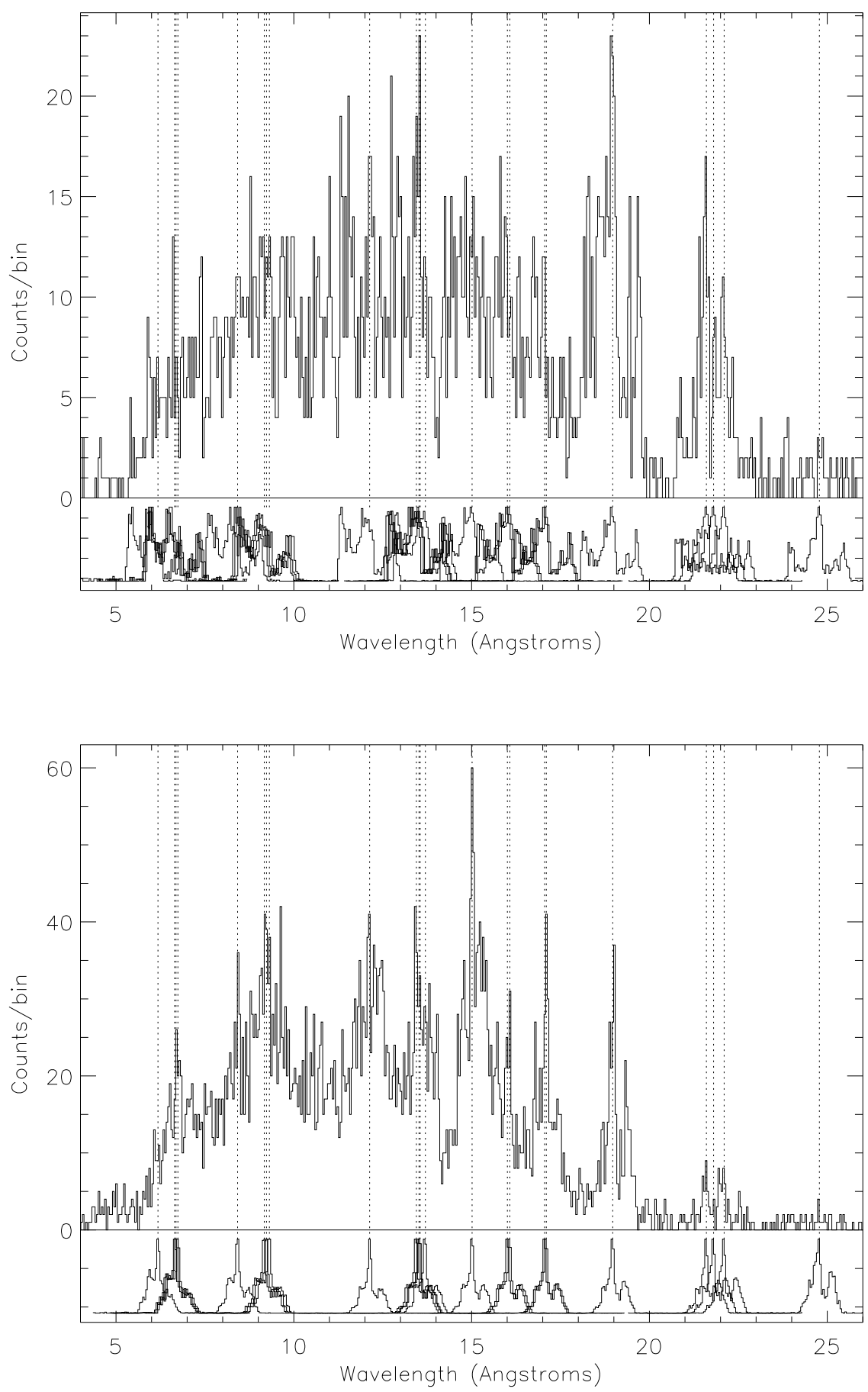

FIGURE 4. "Convolved" spectra (see text for explanation) of selected X-ray bright features in N132D Top: a knot in the uppermost part of the complex of filaments in the interior (it is the topmost filament that appears prominently above the lower vertical marker at $\sim 19 \AA$ in Fig 3 . Bottom: a knot in the bottom outer edge (marked with the lower vertical line in the zeroth order image and in several dispersed images) that appears enhanced at $\sim 17 \AA$ in Fig 3. Spectra are uncorrected for instrumental efficiency. Under each spectrum the effective line shape function is plotted centered at the wavelengths of selected emission lines (marked with vertical dotted lines) which are likely to be strong. 
onto the dispersion axis (so the zeroth order of our example would be projected to a point). Spatial extent in the dispersion direction within each narrow slice (e.g., if the '(' varies in thickness) is not removed, so the projection of the shifted blob onto the dispersion axis becomes the equivalent line shape function. The resulting spectrum is thus the true spectrum convolved with this equivalent line shape function (if there are strong gradients of abundance or temperature across the knot, then of course the line shape function could be different for different spectral lines). The next step, not yet undertaken, is to deconvolve this shape function from the spectrum.

We display in Fig. 4 such "convolved" MEG spectra extracted for two different X-ray bright features in N132D one from the interior (top panel) and the other from the rim (bottom panel) of the SNR image (see figure caption). Below each spectrum, the effective line shape function is plotted repeatedly centered at the wavelengths of selected emission lines that are likely to be strong in N132D. The differences in composition of the two blobs are evident. The interior knot shows strong O viII Ly $\alpha$ emission at $\sim 19 \AA$ and a prominent $\mathrm{O}$ viI triplet at $\sim 22 \AA$, whereas the rim feature shows relatively weak $\mathrm{O}$ emission and a strong Fe XVII 2p-3d lines at 15 and $17 \AA$. We anticipate being able to do a very detailed analysis of the physical conditions and composition of many such features.

\section{CONCLUSIONS}

The remnants Cas A, E0102-72 and N132D, with respective ages of $300 \mathrm{yr}$, 1000-2000 yr, and $3000 \mathrm{yr}$, provide an interesting sequence of SNRs at progressive stages in their evolution. While they have clear differences, such as the presence of O-burning products in the ejecta of Cas A but not of the other two (see the discussion in [2]), they also share significant similarities. All show oxygen rich optical filaments and, with the evidence described here for N132D, all show regions of enhanced oxygen X-ray emission. E0102-72 provides the first clear evidence for a progressive reverse shock that is also implied in the other two SNRs from non-equilibrium analyses.

The two younger members, Cas A and E0102-72, show asymmetric Doppler velocities in the X-ray. Markert et al. [18] discovered the Cas A Doppler velocities and asymmetry using the Einstein Focal Plane Crystal spectrometer, and these were subsequently confirmed and mapped with ASCA [12], with moderate angular resolution. The full velocity range for Cas A is roughly half that seen in E0102, which could well be the result of differences in projection. Thus, the only two young, oxygen-rich SNRs for which such measurements could have been made show the same kind of kinematic behavior. Our approximate model for E0102, like that of [18] for Cas A, suggests that the bright ejecta occupy a partially filled spherical shell in a ring-like geometry (as also suggested by Hughes [13] for E0102). While it is possible that the ejecta themselves were expelled in a ring, it seems more plausible to attribute the geometry to a ring-like distribution of circumstellar 
material which is decelerating and shocking a nearly spherical shell of ejecta, causing it to appear non-spherical. In that case, a significant fraction of the ejecta would still be relatively faint in X-rays. This would fit with the suggestion of Blair et al. [2], who argue that E0102 and N132D may be the remnants of very massive O

stars that underwent substantial mass loss of their outer layers prior to exploding as Type Ib supernovae.

Further study study of these three bright objects is likely to give us new insights into the detailed structure and evolution of the remnants of supernovae in massive stars.

\section{ACKNOWLEDGEMENTS}

We thank the other members of our HETG/CXC group at MIT for their many contributions. This work was supported by NASA contract NAS8-38249 and SAO SV1-61010.

\section{REFERENCES}

1. Canizares, C.R., et al. in preparation.

2. Blair, W.P., Morse, J.A., Raymond, J.C., Kirshner, R.P., Hughes, J.P., Dopita, M.A., Sutherland, R.S., Long, K.S. \& Winkler, P.F., ApJ, 537, 667 (2000).

3. Flanagan, K.A., Canizares, C.R., Davis, D.S., Dewey, D. Houck, J.C., Schattenburg, M.L., these proceedings.

4. Davis, D.S., Flanagan, K.A., Houck, J.C., Allen, G.E., Schulz, N.S., Dewey, D., Schattenburg, \& M.L., these proceedings.

5. Hayashi, I., Koyama, K., Masanobu, O., Miyata, E., Tsunemi, H., Hughes, J.P. \& Petre, R., PASJ, 46, L121 (1994).

6. Gaetz, T.J., Butt, Y.M., Edgar, R.J., Eriksen, K.A., Plucinsky, P.P., Schlegel, E.M. \& Smith, R.K., ApJ, 534, L47 (2000).

7. Hughes, J.P., Rakowski, C.E. \& Decourchelle, A. ApJ, (2000) in press.

8. Eriksen, K.A. et al., these proceedings.

9. Rasmussen, A. et al., these proceedings.

10. Hughes, J.P. \& Helfand, D.J., ApJ, 291, 544 (1985).

11. Tuohy, I.R. \& Dopita, M.A.,ApJ,268, L11 (1983)

12. Holt, S.S., Gotthelf, E.V., Tsunemi, H. \& Negoro, H., PASJ, 46, L151 (1994).

13. Hughes, J.P. 1988, in Supernova Remnants and the Interstellar Medium, ed. R.S. Roger \& T.L. Landecker Cambridge: Cambridge Univ. Press, p. 125.

14. Favata, F., vink, J., Parmar, A.N., Kaastra, J.S., \& Mineo, T., A $6 A$, 324, L45, (1997).

15. Hughes, J.P., Helfand, D.J.Hayashi, I., \& Koyama, K., ApJ, 505, 732 (1998).

16. Hwang, U., Hughes, J.P., Canizares, C.R. \& Markert, T.H., ApJ, 414, 219, (1993).

17. Behar, E. these proceedings.

18. Markert, T.H., Canizares, C.R., Clark, G.W., Winkler, P.F., ApJ, 268, 134 (1983). 